\title{
Applications of Borel distribution series on holomorphic and bi-univalent functions
}

\author{
Abbas Kareem Wanas, Adnan Ghazy Al Amoush
}

\begin{abstract}
In present manuscript, we introduce and study two families $\mathcal{B}_{\Sigma}(\lambda, \delta ; \alpha)$ and $\mathcal{B}_{\Sigma}^{*}(\lambda, \delta ; \beta)$ of holomorphic and bi-univalent functions which involve the Borel distribution series. We establish upper bounds for the initial Taylor-Maclaurin coefficients $\left|a_{2}\right|$ and $\left|a_{3}\right|$ for functions in each of these families. We also point out special cases and consequences of our results.
\end{abstract}

\section{INTRODUCTION}

We indicate by $\mathcal{A}$ the family of functions which are holomorphic in the open unit disk

$$
\mathbb{U}=\{z: z \in \mathbb{C} \text { and }|z|<1\}
$$

and have the following normalized type:

$$
f(z)=z+\sum_{k=2}^{\infty} a_{k} z^{k} .
$$

We also indicate by $\mathcal{S}$ the subclass of $\mathcal{A}$ consisting of functions which are also univalent in $\mathbb{U}$. According to the Koebe one-quarter theorem [8], every function $f \in \mathcal{S}$ has an inverse $f^{-1}$ defined by

$$
f^{-1}(f(z))=z, \quad(z \in \mathbb{U}),
$$

and

where

$$
\left.f\left(f^{-1}(w)\right)=w, q u a d|w|<r_{0}(f) ; r_{0}(f) \geqq \frac{1}{4}\right),
$$

$$
\begin{aligned}
g(w)=f^{-1}(w)= & w-a_{2} w^{2}+\left(2 a_{2}^{2}-a_{3}\right) w^{3} \\
& -\left(5 a_{2}^{3}-5 a_{2} a_{3}+a_{4}\right) w^{4}+\cdots .
\end{aligned}
$$

A function $f \in \mathcal{A}$ is said to be bi-univalent in $\mathbb{U}$ if both $f$ and $f^{-1}$ are univalent in $\mathbb{U}$. Let $\Sigma$ stand for the class of normalized bi-univalent functions

2020 Mathematics Subject Classification. Primary: 30C45; Secondary: 30C50.

Key words and phrases. Holomorphic functions, Bi-univalent functions, Borel distribution series, Coefficient bounds.

Full paper. Received 4 May 2021, revised 22 June 2021, accepted 22 July 2021, available online 23 September 2021. 
in $\mathbb{U}$ given by (1). For a brief historical account and for several interesting examples of functions in the class $\Sigma$, see the pioneering work on this subject by Srivastava et al. [18], which actually revived the study of bi-univalent functions in recent years. From the work of Srivastava et al. [18], we choose to recall here the following examples of functions in the class $\Sigma$ :

$$
\frac{z}{1-z}, \quad-\log (1-z) \text { and } \frac{1}{2} \log \left(\frac{1+z}{1-z}\right) .
$$

We notice that the class $\Sigma$ is not empty. However, the Koebe function is not a member of $\Sigma$.

In a considerably large number of sequels to the aforementioned work of Srivastava et al. [18], several different subclasses of the bi-univalent function class $\Sigma$ were introduced and studied analogously by the many authors (see, for example, [1-7,9-11,13,14,16,17,19-28,30,31]), but only non-sharp estimates on the initial coefficients $\left|a_{2}\right|$ and $\left|a_{3}\right|$ in the Taylor-Maclaurin expansion (1) were obtained in many of these recent papers. The problem to find the general coefficient bounds on the Taylor-Maclaurin coefficients

$$
\left|a_{n}\right|, \quad(n \in \mathbb{N} \backslash\{1,2\} ; \mathbb{N}:=\{1,2,3, \ldots\})
$$

for functions $f \in \Sigma$ is still not completely addressed for many of the subclasses of the bi-univalent function class $\Sigma$ (see, for example, [14,19,21]).

Recently, Srivastava [12] in his survey-cum-expository review article, explored the mathematical application of $q$-calculus, fractional $q$-calculus and fractional $q$-differential operators in Geometric Function Theory.

A discrete random variable $x$ is said to have a Borel distribution, if it takes the values $1,2,3, \ldots$, with the probabilities

$$
\frac{e^{-\delta}}{1 !}, \frac{2 \delta e^{-2 \delta}}{2 !}, \frac{9 \delta^{2} e^{-3 \delta}}{3 !}, \ldots,
$$

respectively, where $\delta$ are called the parameters. Hence

$$
\operatorname{Prob}(x=r)=\frac{(\delta r)^{r-1} e^{-\delta r}}{r !}, \quad(r=1,2,3, \ldots) .
$$

Wanas and Khuttar [29] introduced the following power series whose coefficients are probabilities of the Borel distribution:

$$
\mathcal{M}(\delta, z)=z+\sum_{k=2}^{\infty} \frac{(\delta(k-1))^{k-2} e^{-\delta(k-1)}}{(k-1) !} z^{k}, \quad(z \in \mathbb{U} ; 0<\delta \leqq 1) .
$$

We note by the familiar Ratio Test that the radius of convergence of the above series is infinity.

Now, we considered the linear operator $\mathcal{B}_{\delta}: \mathcal{A} \longrightarrow \mathcal{A}$ which is defined as follows:

$$
\mathcal{B}_{\delta} f(z)=\mathcal{M}(\delta, z) * f(z)=z+\sum_{k=2}^{\infty} \frac{(\delta(k-1))^{k-2} e^{-\delta(k-1)}}{(k-1) !} a_{k} z^{k}, \quad z \in \mathbb{U},
$$


where $(*)$ indicate the Hadamard product (or convolution) of two series.

Very recently, Srivastava and El-Deeb [15] have introduced some applications of the Borel distribution.

We now recall the following lemma that will be used to prove our main results.

Lemma 1 (see [8]). If $h \in \mathcal{P}$, then

$$
\left|c_{k}\right| \leqq 2, \quad(\forall k \in \mathbb{N}),
$$

where $\mathcal{P}$ is the family of all functions $h$, holomorphic in $\mathbb{U}$, for which

$$
\Re(h(z))>0, \quad(z \in \mathbb{U}),
$$

with

$$
h(z)=1+c_{1} z+c_{2} z^{2}+\cdots, \quad(z \in \mathbb{U}) .
$$

\section{Coefficient estimates for the Bi-Univalent} FUNCTION CLASS $\mathcal{B}_{\Sigma}(\lambda, \delta ; \alpha)$

In this section, we first define the bi-univalent function class $\mathcal{B}_{\Sigma}(\lambda, \delta ; \alpha)$.

Definition 1. A function $f \in \Sigma$, given by (1), in said to be the bi-univalent function class $\mathcal{B}_{\Sigma}(\lambda, \delta ; \alpha)$ if it satisfies the following conditions:

$$
\left|\arg \left(1+\frac{z\left(\mathcal{B}_{\delta} f(z)\right)^{\prime}}{\mathcal{B}_{\delta} f(z)}+\frac{z\left(\mathcal{B}_{\delta} f(z)\right)^{\prime \prime}}{\left(\mathcal{B}_{\delta} f(z)\right)^{\prime}}-\frac{\lambda z^{2}\left(\mathcal{B}_{\delta} f(z)\right)^{\prime \prime}+z\left(\mathcal{B}_{\delta} f(z)\right)^{\prime}}{\lambda z\left(\mathcal{B}_{\delta} f(z)\right)^{\prime}+(1-\lambda) \mathcal{B}_{\delta} f(z)}\right)\right|<\frac{\alpha \pi}{2}
$$

and

$$
\left|\arg \left(1+\frac{w\left(\mathcal{B}_{\delta} g(w)\right)^{\prime}}{\mathcal{B}_{\delta} g(w)}+\frac{w\left(\mathcal{B}_{\delta} g(w)\right)^{\prime \prime}}{\left(\mathcal{B}_{\delta} g(w)\right)^{\prime}}-\frac{\lambda w^{2}\left(\mathcal{B}_{\delta} g(w)\right)^{\prime \prime}+w\left(\mathcal{B}_{\delta} g(w)\right)^{\prime}}{\lambda w\left(\mathcal{B}_{\delta} g(w)\right)^{\prime}+(1-\lambda) \mathcal{B}_{\delta} g(w)}\right)\right|<\frac{\alpha \pi}{2},
$$

where

$$
z, w \in \mathbb{U}, \quad 0<\alpha \leqq 1, \quad 0 \leqq \lambda \leqq 1 \quad \text { and } \quad 0<\delta \leqq 1,
$$

and $g=f^{-1}$ is given by (2).

In particular, if we choose $\lambda=1$ in Definition 1 , the family $\mathcal{B}_{\Sigma}(\lambda, \delta ; \alpha)$ reduces to the family $S_{\Sigma}(\delta ; \alpha)$ of bi-starlike functions which satisfying the following conditions

$$
\left|\arg \left(\frac{z\left(\mathcal{B}_{\delta} f(z)\right)^{\prime}}{\mathcal{B}_{\delta} f(z)}\right)\right|<\frac{\alpha \pi}{2}
$$

and

$$
\left|\arg \left(\frac{w\left(\mathcal{B}_{\delta} g(w)\right)^{\prime}}{\mathcal{B}_{\delta} g(w)}\right)\right|<\frac{\alpha \pi}{2} .
$$

If we choose $\lambda=0$ in Definition 1 , the family $\mathcal{B}_{\Sigma}(\lambda, \delta ; \alpha)$ reduces to the family $\mathcal{K}_{\Sigma}(\delta ; \alpha)$ of bi-convex functions which satisfying the following conditions:

$$
\left|\arg \left(1+\frac{z\left(\mathcal{B}_{\delta} f(z)\right)^{\prime \prime}}{\left(\mathcal{B}_{\delta} f(z)\right)^{\prime}}\right)\right|<\frac{\alpha \pi}{2}
$$


and

$$
\left|\arg \left(1+\frac{w\left(\mathcal{B}_{\delta} g(w)\right)^{\prime \prime}}{\left(\mathcal{B}_{\delta} g(w)\right)^{\prime}}\right)\right|<\frac{\alpha \pi}{2} .
$$

Our first main result is asserted by Theorem 1 below.

Theorem 1. Let the function $f \in \mathcal{B}_{\Sigma}(\lambda, \delta ; \alpha)(0<\alpha \leqq 1 ; 0 \leqq \lambda \leqq 1 ; 0<$ $\delta \leqq 1$ ) be given by (1). Then

$$
\left|a_{2}\right| \leqq \frac{2 \alpha}{\sqrt{\left|2 \alpha e^{-2 \delta}\left[(\lambda+1)^{2}+2 \delta(3-2 \lambda)-5\right]+(1-\alpha)(2-\lambda)^{2} e^{-2 \delta}\right|}}
$$

and

$$
\left|a_{3}\right| \leqq \frac{4 \alpha^{2} e^{2 \delta}}{(2-\lambda)^{2}}+\frac{\alpha e^{2 \delta}}{\delta(3-2 \lambda)} .
$$

Proof. In light of the conditions (3) and (4), we have

(5) $1+\frac{z\left(\mathcal{B}_{\delta} f(z)\right)^{\prime}}{\mathcal{B}_{\delta} f(z)}+\frac{z\left(\mathcal{B}_{\delta} f(z)\right)^{\prime \prime}}{\left(\mathcal{B}_{\delta} f(z)\right)^{\prime}}-\frac{\lambda z^{2}\left(\mathcal{B}_{\delta} f(z)\right)^{\prime \prime}+z\left(\mathcal{B}_{\delta} f(z)\right)^{\prime}}{\lambda z\left(\mathcal{B}_{\delta} f(z)\right)^{\prime}+(1-\lambda) \mathcal{B}_{\delta} f(z)}=[p(z)]^{\alpha}$

and

(6) $1+\frac{w\left(\mathcal{B}_{\delta} g(w)\right)^{\prime}}{\mathcal{B}_{\delta} g(w)}+\frac{w\left(\mathcal{B}_{\delta} g(w)\right)^{\prime \prime}}{\left(\mathcal{B}_{\delta} g(w)\right)^{\prime}}-\frac{\lambda w^{2}\left(\mathcal{B}_{\delta} g(w)\right)^{\prime \prime}+w\left(\mathcal{B}_{\delta} g(w)\right)^{\prime}}{\lambda w\left(\mathcal{B}_{\delta} g(w)\right)^{\prime}+(1-\lambda) \mathcal{B}_{\delta} g(w)}=[q(w)]^{\alpha}$,

where $g=f^{-1}$ and the functions $p, q \in \mathcal{P}$ have the following series representations:

$$
p(z)=1+p_{1} z+p_{2} z^{2}+p_{3} z^{3}+\cdots
$$

and

$$
q(w)=1+q_{1} w+q_{2} w^{2}+q_{3} w^{3}+\cdots .
$$

By comparing the corresponding coefficients of (5) and (6), we find that

$$
(2-\lambda) e^{-\delta} a_{2}=\alpha p_{1},
$$

and

$$
\begin{aligned}
2 \delta(3-2 \lambda) e^{-2 \delta} a_{3}- & \left(5-(\lambda+1)^{2}\right) e^{-2 \delta} a_{2}^{2}=\alpha p_{2}+\frac{\alpha(\alpha-1)}{2} p_{1}^{2}, \\
& -(2-\lambda) e^{-\delta} a_{2}=\alpha q_{1}
\end{aligned}
$$

Thus, by using (9) and (11), we conclude that

$$
p_{1}=-q_{1}
$$

and

$$
2(2-\lambda)^{2} e^{-2 \delta} a_{2}^{2}=\alpha^{2}\left(p_{1}^{2}+q_{1}^{2}\right) .
$$


If we add (10) to (12), we obtain

$$
2 e^{-2 \delta}\left[(\lambda+1)^{2}+2 \delta(3-2 \lambda)-5\right] a_{2}^{2}=\alpha\left(p_{2}+q_{2}\right)+\frac{\alpha(\alpha-1)}{2}\left(p_{1}^{2}+q_{1}^{2}\right) .
$$

Substituting the value of $p_{1}^{2}+q_{1}^{2}$ from (14) into the right-hand side of (15), and after some computations, we deduce that

$$
a_{2}^{2}=\frac{\alpha^{2}\left(p_{2}+q_{2}\right)}{2 \alpha e^{-2 \delta}\left[(\lambda+1)^{2}+2 \delta(3-2 \lambda)-5\right]+(1-\alpha)(2-\lambda)^{2} e^{-2 \delta}} .
$$

By taking the moduli of both sides of (16) and applying the Lemma 1 for the coefficients $p_{2}$ and $q_{2}$, we have

$$
\left|a_{2}\right| \leqq \frac{2 \alpha}{\sqrt{\left|2 \alpha e^{-2 \delta}\left[(\lambda+1)^{2}+2 \delta(3-2 \lambda)-5\right]+(1-\alpha)(2-\lambda)^{2} e^{-2 \delta}\right|}} .
$$

Next, in order to determinate the bound on $\left|a_{3}\right|$, by subtracting (12) from (10), we get

$$
4 \delta(3-2 \lambda) e^{-2 \delta}\left(a_{3}-a_{2}^{2}\right)=\alpha\left(p_{2}-q_{2}\right)+\frac{\alpha(\alpha-1)}{2}\left(p_{1}^{2}-q_{1}^{2}\right) .
$$

Now, upon substituting the value of $a_{2}^{2}$ from (14) into (17) and using (13), we deduce that

$$
a_{3}=\frac{\alpha^{2}\left(p_{1}^{2}+q_{1}^{2}\right)}{2(2-\lambda)^{2} e^{-2 \delta}}+\frac{\alpha\left(p_{2}-q_{2}\right)}{4 \delta(3-2 \lambda) e^{-2 \delta}} .
$$

Finally, by taking the moduli on both sides of (18) and applying the Lemma 1 once again for the coefficients $p_{1}, p_{2}, q_{1}$ and $q_{2}$, it follows that

$$
\left|a_{3}\right| \leqq \frac{4 \alpha^{2} e^{2 \delta}}{(2-\lambda)^{2}}+\frac{\alpha e^{2 \delta}}{\delta(3-2 \lambda)} .
$$

This completes the proof of Theorem 1 .

Putting $\lambda=1$ in Theorem 1, we state:

Corollary 1. For $0<\alpha \leqq 1$ and $0<\delta \leqq 1$, let the function $f \in S_{\Sigma}(\delta ; \alpha)$ be given by (1). Then

$$
\left|a_{2}\right| \leqq \frac{2 \alpha}{\sqrt{\left|2 \alpha(2 \delta-1) e^{-2 \delta}+(1-\alpha) e^{-2 \delta}\right|}}
$$

and

$$
\left|a_{3}\right| \leqq 4 \alpha^{2} e^{2 \delta}+\frac{1}{\delta} \alpha e^{2 \delta} .
$$

Putting $\lambda=0$ in Theorem 1, we state: 
Corollary 2. For $0<\alpha \leqq 1$ and $0<\delta \leqq 1$, let the function $f \in \mathcal{K}_{\Sigma}(\delta ; \alpha)$ be given by (1). Then

$$
\left|a_{2}\right| \leqq \frac{\alpha}{\sqrt{\left|\alpha e^{-2 \delta}(3 \delta-2)+(1-\alpha) e^{-2 \delta}\right|}}
$$

and

$$
\left|a_{3}\right| \leqq \alpha^{2} e^{2 \delta}+\frac{1}{3 \delta} \alpha e^{2 \delta}
$$

\section{Coefficient estimates for the Bi-Univalent FUNCTION CLASS $\mathcal{B}_{\Sigma}^{*}(\lambda, \delta ; \beta)$}

In this section, we first define the bi-univalent function class $\mathcal{B}_{\Sigma}^{*}(\lambda, \delta ; \beta)$.

Definition 2. A function $f \in \Sigma$, given by (1), is said to be in the biunivalent function class $\mathcal{B}_{\Sigma}^{*}(\lambda, \delta ; \beta)$ if it satisfies the following conditions:

$$
\Re\left\{1+\frac{z\left(\mathcal{B}_{\delta} f(z)\right)^{\prime}}{\mathcal{B}_{\delta} f(z)}+\frac{z\left(\mathcal{B}_{\delta} f(z)\right)^{\prime \prime}}{\left(\mathcal{B}_{\delta} f(z)\right)^{\prime}}-\frac{\lambda z^{2}\left(\mathcal{B}_{\delta} f(z)\right)^{\prime \prime}+z\left(\mathcal{B}_{\delta} f(z)\right)^{\prime}}{\lambda z\left(\mathcal{B}_{\delta} f(z)\right)^{\prime}+(1-\lambda) \mathcal{B}_{\delta} f(z)}\right\}>\beta
$$

and

$$
\Re\left\{1+\frac{w\left(\mathcal{B}_{\delta} g(w)\right)^{\prime}}{\mathcal{B}_{\delta} g(w)}+\frac{w\left(\mathcal{B}_{\delta} g(w)\right)^{\prime \prime}}{\left(\mathcal{B}_{\delta} g(w)\right)^{\prime}}-\frac{\lambda w^{2}\left(\mathcal{B}_{\delta} g(w)\right)^{\prime \prime}+w\left(\mathcal{B}_{\delta} g(w)\right)^{\prime}}{\lambda w\left(\mathcal{B}_{\delta} g(w)\right)^{\prime}+(1-\lambda) \mathcal{B}_{\delta} g(w)}\right\}>\beta,
$$

where

$$
z, w \in \mathbb{U}, \quad 0 \leqq \beta<1, \quad 0 \leqq \lambda \leqq 1 \quad \text { and } \quad 0<\delta \leqq 1,
$$

and $g=f^{-1}$ is given by (2).

In particular, if we choose $\lambda=1$ in Definition 2 , the family $\mathcal{B}_{\Sigma}^{*}(\lambda, \delta ; \beta)$ reduces to the family $S_{\Sigma}^{*}(\delta ; \beta)$ of bi-starlike functions which satisfying the following conditions

$$
\Re\left\{\frac{z\left(\mathcal{B}_{\delta} f(z)\right)^{\prime}}{\mathcal{B}_{\delta} f(z)}\right\}>\beta
$$

and

$$
\Re\left\{\frac{w\left(\mathcal{B}_{\delta} g(w)\right)^{\prime}}{\mathcal{B}_{\delta} g(w)}\right\}>\beta .
$$

Also, if we choose $\lambda=0$ in Definition 2 , the family $\mathcal{B}_{\Sigma}^{*}(\lambda, \delta ; \beta)$ reduces to the family $\mathcal{K}_{\Sigma}^{*}(\delta ; \beta)$ of bi-convex functions which satisfying the following conditions

$$
\Re\left\{1+\frac{z\left(\mathcal{B}_{\delta} f(z)\right)^{\prime \prime}}{\left(\mathcal{B}_{\delta} f(z)\right)^{\prime}}\right\}>\beta
$$

and

$$
\Re\left\{1+\frac{w\left(\mathcal{B}_{\delta} g(w)\right)^{\prime \prime}}{\left(\mathcal{B}_{\delta} g(w)\right)^{\prime}}\right\}>\beta .
$$

Our second main result is asserted by Theorem 2 below. 
Theorem 2. Let the function $f \in \mathcal{B}_{\Sigma}^{*}(\lambda, \delta ; \beta)(0 \leqq \beta<1 ; 0 \leqq \lambda \leqq 1 ; 0<$ $\delta \leqq 1$ ) be given by (1). Then

$$
\left|a_{2}\right| \leqq \sqrt{\frac{2(1-\beta)}{\left|e^{-2 \delta}\left[(\lambda+1)^{2}+2 \delta(3-2 \lambda)-5\right]\right|}}
$$

and

$$
\left|a_{3}\right| \leqq \frac{4(1-\beta)^{2} e^{2 \delta}}{(2-\lambda)^{2}}+\frac{(1-\beta) e^{2 \delta}}{\delta(3-2 \lambda)} .
$$

Proof. In view of the conditions (19) and (20), there exist the functions $p, q \in \mathcal{P}$ such that

$$
\begin{aligned}
1+ & \frac{z\left(\mathcal{B}_{\delta} f(z)\right)^{\prime}}{\mathcal{B}_{\delta} f(z)}+\frac{z\left(\mathcal{B}_{\delta} f(z)\right)^{\prime \prime}}{\left(\mathcal{B}_{\delta} f(z)\right)^{\prime}} \\
& -\frac{\lambda z^{2}\left(\mathcal{B}_{\delta} f(z)\right)^{\prime \prime}+z\left(\mathcal{B}_{\delta} f(z)\right)^{\prime}}{\lambda z\left(\mathcal{B}_{\delta} f(z)\right)^{\prime}+(1-\lambda) \mathcal{B}_{\delta} f(z)}=\beta+(1-\beta) p(z)
\end{aligned}
$$

and

$$
\begin{aligned}
1+ & \frac{w\left(\mathcal{B}_{\delta} g(w)\right)^{\prime}}{\mathcal{B}_{\delta} g(w)}+\frac{w\left(\mathcal{B}_{\delta} g(w)\right)^{\prime \prime}}{\left(\mathcal{B}_{\delta} g(w)\right)^{\prime}} \\
& -\frac{\lambda w^{2}\left(\mathcal{B}_{\delta} g(w)\right)^{\prime \prime}+w\left(\mathcal{B}_{\delta} g(w)\right)^{\prime}}{\lambda w\left(\mathcal{B}_{\delta} g(w)\right)^{\prime}+(1-\lambda) \mathcal{B}_{\delta} g(w)}=\beta+(1-\beta) q(w),
\end{aligned}
$$

where $g=f^{-1}$ and the functions $p, q \in \mathcal{P}$ have the series expansions given by (7) and (8), respectively. Thus, by comparing the corresponding coefficients in $(21)$ and (22), we get

$$
\begin{gathered}
(2-\lambda) e^{-\delta} a_{2}=(1-\beta) p_{1}, \\
2 \delta(3-2 \lambda) e^{-2 \delta} a_{3}-\left(5-(\lambda+1)^{2}\right) e^{-2 \delta} a_{2}^{2}=(1-\beta) p_{2}, \\
-(2-\lambda) e^{-\delta} a_{2}=(1-\beta) q_{1}
\end{gathered}
$$

and

$$
2 \delta(3-2 \lambda) e^{-2 \delta}\left(2 a_{2}^{2}-a_{3}\right)-\left(5-(\lambda+1)^{2}\right) e^{-2 \delta} a_{2}^{2}=(1-\beta) q_{2} .
$$

We now find from (23)) and (25)) that

$$
p_{1}=-q_{1}
$$

and

$$
2(2-\lambda)^{2} e^{-2 \delta} a_{2}^{2}=(1-\beta)^{2}\left(p_{1}^{2}+q_{1}^{2}\right) .
$$

By adding (24) and (26), we obtain

$$
2 e^{-2 \delta}\left[(\lambda+1)^{2}+2 \delta(3-2 \lambda)-5\right] a_{2}^{2}=(1-\beta)\left(p_{2}+q_{2}\right) .
$$


Consequently, we have

$$
a_{2}^{2}=\frac{(1-\beta)\left(p_{2}+q_{2}\right)}{2 e^{-2 \delta}\left[(\lambda+1)^{2}+2 \delta(3-2 \lambda)-5\right]} .
$$

Next, by applying the Lemma 1 for the coefficients $p_{2}$ and $q_{2}$, we deduce that

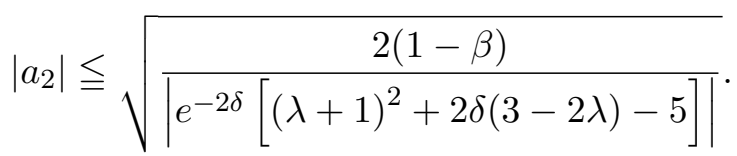

In order to determinate the bound on $\left|a_{3}\right|$, by subtracting (26) from (24), we get

$$
4 \delta(3-2 \lambda) e^{-2 \delta}\left(a_{3}-a_{2}^{2}\right)=(1-\beta)\left(p_{2}-q_{2}\right)
$$

or, equivalently,

$$
a_{3}=a_{2}^{2}+\frac{(1-\beta)\left(p_{2}-q_{2}\right)}{4 \delta(3-2 \lambda) e^{-2 \delta}} .
$$

Substituting the value of $a_{2}^{2}$ from (27) into (28), it follows that

$$
a_{3}=\frac{(1-\beta)^{2}\left(p_{1}^{2}+q_{1}^{2}\right)}{2(2-\lambda)^{2} e^{-2 \delta}}+\frac{(1-\beta)\left(p_{2}-q_{2}\right)}{4 \delta(3-2 \lambda) e^{-2 \delta}} .
$$

Finally, by applying the Lemma 1 once again for the coefficients $p_{1}, p_{2}, q_{1}$ and $q_{2}$, we get

$$
\left|a_{3}\right| \leqq \frac{4(1-\beta)^{2} e^{2 \delta}}{(2-\lambda)^{2}}+\frac{(1-\beta) e^{2 \delta}}{\delta(3-2 \lambda)} .
$$

We have thus completed the proof of Theorem 2 .

Putting $\lambda=1$ in Theorem 2, we state:

Corollary 3. For $0 \leqq \beta<1$ and $0<\delta \leqq 1$, let $f \in S_{\Sigma}^{*}(\delta ; \beta)$ be given by (1). Then

$$
\left|a_{2}\right| \leqq \sqrt{\frac{2(1-\beta)}{\left|(2 \delta-1) e^{-2 \delta}\right|}}
$$

and

$$
\left|a_{3}\right| \leqq 4(1-\beta)^{2} e^{2 \delta}+\frac{1}{\delta}(1-\beta) e^{2 \delta} .
$$

Putting $\lambda=0$ in Theorem 2, we state:

Corollary 4. For $0 \leqq \beta<1$ and $0<\delta \leqq 1$, let $f \in \mathcal{K}_{\Sigma}^{*}(\delta ; \beta)$ be given by (1). Then

$$
\left|a_{2}\right| \leqq \sqrt{\frac{1-\beta}{\left|(3 \delta-2) e^{-2 \delta}\right|}}
$$

and

$$
\left|a_{3}\right| \leqq(1-\beta)^{2} e^{2 \delta}+\frac{1}{3 \delta}(1-\beta) e^{2 \delta} \text {. }
$$




\section{Acknowledgments}

The authors would like to thank the referee(s) for their helpful comments and suggestions.

\section{REFERENCES}

[1] C. Abirami, N. Magesh, J. Yamini, Initial bounds for certain classes of bi-univalent functions defined by Horadam polynomials, Abstract and Applied Analysis, (2020), Article ID: 7391058, 1-8.

[2] E. A. Adegani, S. Bulut, A. A. Zireh, Coefficient estimates for a subclass of analytic bi-univalent functions, Bulletin of the Korean Mathematical Society, 55 (2) (2018), 405-413.

[3] A. G. Alamoush, Certain subclasses of bi-univalent functions involving the Poisson distribution associated with Horadam polynomials, Malaya Journal of Matematik, 7 (2019), 618-624.

[4] A. G. Alamoush, Coefficient estimates for a new subclasses of lambda-pseudo biunivalent functions with respect to symmetrical points associated with the Horadam Polynomials, Turkish Journal of Mathematics, 3 (2019), 2865-2875.

[5] A. G. Alamoush, On a subclass of bi-univalent functions associated to Horadam polynomials, International Journal of Open Problems in Complex Analysis, 12 (2020), $58-66$.

[6] A. G. Alamoush, On subclass of analytic bi-close-to-convex functions, International Journal of Open Problems in Complex Analysis, 13 (2021), 10-18.

[7] S. Bulut, A. K. Wanas, Coefficient estimates for families of bi-univalent functions defined by Ruscheweyh derivative operator, Mathematica Moravica, 25 (1) (2021), $71-80$.

[8] P. L. Duren, Univalent Functions, Grundlehren der Mathematischen Wissenschaften, Band 259, Springer Verlag, New York, Berlin, Heidelberg and Tokyo, 1983.

[9] H. Ö. Güney, G. Murugusundaramoorthy, J. Sokól, Subclasses of bi-univalent functions related to shell-like curves connected with Fibonacci numbers, Acta Universitatis Sapientiae Mathematica, 10 (2018), 70-84.

[10] N. Magesh, S. Bulut, Chebyshev polynomial coefficient estimates for a class of analytic bi-univalent functions related to pseudo-starlike functions, Afrika Matematika, 29 (1-2) (2018), 203-209.

[11] B. Şeker, On a new subclass of bi-univalent functions defined by using Salagean operator, Turkish Journal of Mathematics, 42 (2018), 2891-2896.

[12] H. M. Srivastava, Operators of basic (or q-) calculus and fractional q-calculus and their applications in geometric function theory of complex analysis, Iranian Journal of Science and Technology, Transactions A: Science, 44 (2020), 327-344.

[13] H. M. Srivastava, Ş. Altınkaya, S. Yalçin, Certain subclasses of bi-univalent functions associated with the Horadam polynomials, Iranian Journal of Science and Technology, Transactions A: Science, 43 (2019), 1873-1879. 
[14] H. M. Srivastava, S. S. Eker, S. G. Hamidi, J. M. Jahangiri, Faber polynomial coefficient estimates for bi-univalent functions defined by the Tremblay fractional derivative operator, Bulletin of the Iranian Mathematical Society, 44 (1) (2018), 149-157.

[15] H. M. Srivastava, S. M. El-Deeb, Fuzzy differential subordinations based upon the Mittag-Leffler type Borel distribution, Symmetry, 13 (2021), Article ID: 1023, 1-15.

[16] H. M. Srivastava, S. Gaboury, F. Ghanim, Coefficient estimates for some general subclasses of analytic and bi-univalent functions, Afrika Matematika, 28 (2017), 693706.

[17] H. M. Srivastava, S. Gaboury, F. Ghanim, Coefficient estimates for a general subclass of analytic and bi-univalent functions of the Ma-Minda type, Revista de la Real Academia de Ciencias Exactas, Físicas y Naturales. Serie A. Matemáticas (RACSAM), 112 (2018), 1157-1168.

[18] H. M. Srivastava, A. K. Mishra, P. Gochhayat, Certain subclasses of analytic and bi-univalent functions, Applied Mathematics Letters, 23 (2010), 1188-1192.

[19] H. M. Srivastava, A. Motamednezhad, E. A. Adegani, Faber polynomial coefficient estimates for bi-univalent functions defined by using differential subordination and a certain fractional derivative operator, Mathematics, 8 (2020), Article ID: 172, 1-12.

[20] H. M. Srivastava, A. Motamednezhad, S. Salehian, Coefficients of a comprehensive subclass of meromorphic bi-univalent functions associated with the Faber polynomial expansion, Axioms, 10 (2021), Article ID: 27, 1-13.

[21] H. M. Srivastava, F. M. Sakar, H. Ö. Güney, Some general coefficient estimates for a new class of analytic and bi-univalent functions defined by a linear combination, Filomat, 32 (2018), 1313-1322.

[22] H. M. Srivastava, A. K. Wanas, Initial Maclaurin coefficient bounds for new subclasses of analytic and $m$-fold symmetric bi-univalent functions defined by a linear combination, Kyungpook Mathematical Journal, 59 (3) (2019), 493-503.

[23] H. M. Srivastava, A. K. Wanas, G. Murugusundaramoorthy, Certain family of biunivalent functions associated with Pascal distribution series based on Horadam polynomials, Surveys in Mathematics and its Applications, 16 (2021), 193-205.

[24] S. R. Swamy, A. K. Wanas, Y. Sailaja, Some special families of holomorphic and Sălăgean type bi-univalent functions associated with (m,n)-Lucas polynomials, Communications in Mathematics and Applications, 11 (4) (2020), 563-574.

[25] A. K. Wanas, Applications of (M,N)-Lucas polynomials for holomorphic and biunivalent functions, Filomat, 34 (10) (2020), 3361-3368.

[26] A. K. Wanas, Coefficient estimates for Bazilevič functions of bi-prestarlike functions, Miskolc Mathematical Notes, 21 (2) (2020), 1031-1040.

[27] A. K. Wanas, Applications of Chebyshev polynomials on $\lambda$-pseudo bi-starlike and $\lambda$-pseudo bi-convex functions with respect to symmetrical points, TWMS Journal of Applied and Engineering Mathematics, 10 (3) (2020), 568-573.

[28] A. K. Wanas, A. L. Alina, Applications of Horadam polynomials on Bazilevič biunivalent function satisfying subordinate conditions, Journal of Physics: Conference Series, 1294 (2019), 1-6. 
[29] A. K. Wanas, J. A. Khuttar, Applications of Borel distribution series on analytic functions, Earthline Journal of Mathematical Sciences, 4 (2020), 71-82.

[30] A. K. Wanas, A. H. Majeed, On subclasses of analytic and $m$-fold symmetric biunivalent functions, Iranian Journal of Mathematical Sciences and Informatics, 15 (2) (2020), 51-60.

[31] A. K. Wanas, H. Tang, Initial Coefficient estimates for a classes of $m$-fold symmetric bi-univalent functions involving Mittag-Leffler function, Mathematica Moravica, 24 (2) (2020), 51-61.

\author{
Abbas Kareem Wanas \\ Department of Mathematics \\ College of Science \\ University of Al-QAdisiyah \\ IRAQ \\ E-mail address: abbas.kareem.w@qu.edu.iq
}

\author{
Adnan Ghazy Al Amoush \\ Faculty of Science \\ TAIBAH UNIVERSITY \\ Saudi Arabia \\ E-mail address: adnan_omoush@yahoo.com
}

